\title{
A Multilingual Evaluation of Three Spelling Normalisation Methods for Historical Text
}

\author{
Eva Pettersson ${ }^{1,2}$, Beáta Megyesi ${ }^{1}$ and Joakim Nivre ${ }^{1}$ \\ (1) Department of Linguistics and Philology \\ Uppsala University \\ (2) Swedish National Graduate School \\ of Language Technology \\ firstname. lastnamedlingfil.uu.se
}

\begin{abstract}
We present a multilingual evaluation of approaches for spelling normalisation of historical text based on data from five languages: English, German, Hungarian, Icelandic, and Swedish. Three different normalisation methods are evaluated: a simplistic filtering model, a Levenshteinbased approach, and a character-based statistical machine translation approach. The evaluation shows that the machine translation approach often gives the best results, but also that all approaches improve over the baseline and that no single method works best for all languages.
\end{abstract}

\section{Introduction}

Language technology for historical text is a field of research imposing a variety of challenges. Nevertheless, there is an increasing need for natural language processing (NLP) tools adapted to historical texts, as an aid for researchers in the humanities field. For example, the historians in the Gender and Work project are studying what men and women did for a living in the Early Modern Swedish society (Ågren et al., 2011). In this project, researchers have found that the most important words in revealing this information are verbs such as fishing, selling etc. Instead of manually going through written sources from this time period, it is therefore assumed that an NLP tool that automatically searches through a number of historical documents and presents the contained verbs (and possibly their complements), would make the process of finding relevant text passages more effective.

A major challenge in developing language technology for historical text is that historical language often is under-resourced with regard to annotated data needed for training NLP tools. This prob- lem is further aggravated by the fact that historical texts may refer to texts from a long period of time, during which language has changed. NLP tools trained on 13th century texts may thus not perform well on texts from the 18th century. Furthermore, historical language usually shows a substantial variation in spelling and grammar between different genres, different authors and even within the same text written by the same author, due to the lack of spelling conventions.

To deal with the limited resources and the high degree of spelling variation, one commonly applied approach is to automatically normalise the original spelling to a more modern spelling, before applying the NLP tools. This way, NLP tools available for the modern language may be used to analyse historical text. Even though there may be structural differences as well between historical and modern language, spelling is the most striking difference. Moreover, language technology tools such as taggers often to some degree rely on statistics on word form n-grams and token frequencies, implying that spelling modernisation is an important step for improving the performance of such tools when applied to historical text. This paper presents an evaluation of three approaches to spelling normalisation: 1) a filtering approach based on corpus data, 2) an approach based on Levenshtein edit distance, and 3) an approach implementing character-based statistical machine translation (SMT) techniques. These approaches have previously solely been evaluated in isolation, without comparison to each other, and for one or two languages only. We compare the results of the different methods in a multilingual evaluation including five languages, and we show that all three approaches have a positive impact on normalisation accuracy as compared to the baseline. There is no single method that yields the highest normalisation accuracy for all languages, but for four out of five languages within the scope 
of our study, the SMT-based approach gives the best results.

\section{Related Work}

Spelling normalisation of historical text has previously been approached using techniques such as dictionary lookup, edit distance calculations, and machine translation.

Rayson et al. (2005) tried an approach based on dictionary lookup, where a mapping scheme from historical to modern spelling for 16th to 19th century English texts was manually created, resulting in the VARD tool (VARiant Detector) comprising 45,805 entries. The performance of the normalisation tool was evaluated on a set of 17 th century texts, and compared to the performance of modern spell checkers on the same text. The results showed that between a third and a half of all tokens (depending on which test text was used) were correctly normalised by both VARD and MS Word, whereas approximately one third of the tokens were correctly normalised only when using VARD. The percentage of tokens correctly normalised only by MS Word was substantially lower; approximately $6 \%$. VARD was later further developed into VARD2, combining the original word list with data-driven techniques in the form of phonetic matching against a modern dictionary, and letter replacement rules based on common spelling variation patterns (Baron and Rayson, 2008).

Jurish (2008) argued that due to the lack of orthographic conventions, spelling generally reflects the phonetic form of the word to a higher degree in historical text. Furthermore, it is assumed that phonetic properties are less resistant to diachronic change than orthography. Accordingly, Jurish explored the idea of comparing the similarity between phonetic forms rather than orthographic forms. For grapheme-to-phoneme conversion, a module of the IMS German Festival textto-speech system (Black and Taylor, 1997) was used, with a rule-set adapted to historical word forms. Evaluation was performed on a corpus of historical German verse quotations extracted from Deutsches Wörterbuch, containing 5,491,982 tokens (318,383 types). Without normalisation, approximately $84 \%$ of the tokens were recognised by a morphological analyser. After normalisation, $92 \%$ of the tokens were recognised. Adding lemma-based heuristics, coverage increased further to $94 \%$ of the tokens.
A Levenshtein similarity approach to normalisation was presented by Bollmann et al. (2011) for Early New High German, where Levenshteinbased normalisation rules were automatically derived from a word-aligned parallel corpus consisting of the Martin Luther Bible in its 1545 edition and its 1892 version, respectively. Using this normalisation technique, the proportion of words with a spelling identical to the modern spelling increased from $65 \%$ in the original text to $91 \%$ in the normalised text. This normalisation method was further evaluated by Bollmann (2013), comparing the performance of the RFTagger applied to historical text before and after normalisation. For every evaluation text, the tagger was trained on between 100 and 1,000 manually normalised tokens, and evaluated on the remaining tokens in the same text. For one manuscript from the 15th century, tagging accuracy was improved from approximately $29 \%$ to $78 \%$ using this method.

Another Levenshtein-based approach to normalisation was presented by Pettersson et al. (2013b), using context-sensitive, weighted edit distance calculations combined with compound splitting. This method requires no annotated historical training data, since normalisation candidates are extracted by Levenshtein comparisons between the original historical word form and present-day dictionary entries. However, if a corpus of manually normalised historical text is available, this can optionally be included for dictionary lookup and weighted Levenshtein calculations, improving precision. This technique was evaluated for Early Modern Swedish, and in the best setting, the proportion of words in the historical text with a spelling identical to the modern gold standard spelling increased from $64.6 \%$ to $86.9 \%$.

Pettersson et al. (2013a) treated the normalisation task as a translation problem, using characterbased SMT techniques in the spelling normalisation process. With the SMT-based approach, the proportion of tokens in the historical text with a spelling identical to the modern gold standard spelling increased from $64.6 \%$ to $92.3 \%$ for Early Modern Swedish, and from $64.8 \%$ to $83.9 \%$ for 15 th century Icelandic. It was also shown that normalisation had a positive effect on subsequent tagging and parsing.

Language technology for historical text also has a lot in common with adaptation of NLP tools 
for handling present-day SMS messages and microblog text such as Twitter. In both genres there is a high degree of spelling variation, ad hoc abbreviations and ungrammatical structures imposing the problem of data sparseness. Similar methods for spelling normalisation may thus be used for both tasks. Han and Baldwin (2011) presented a method for normalising SMS and Twitter text based on morphophonemic similarity, combining lexical edit distance, phonemic edit distance, prefix substring, suffix substring, and the longest common subsequence. Context was taken into account by means of dependency structures generated by the Stanford Parser applied to a corpus of New York Times articles. In the best setting, a token-level F-score of $75.5 \%$ and $75.3 \%$ was reported for SMS messages and Twitter texts respectively.

\section{Approaches}

\subsection{The Filtering Approach}

The filtering approach presupposes access to a parallel training corpus of token pairs with historical word forms mapped to their modernised spelling. In the normalisation process, whenever a token is encountered that also occurred in the training data, the most frequent modern spelling associated with that token in the training corpus is chosen for normalisation. Other tokens are left unchanged.

\subsection{The Levenshtein-based Approach}

The Levenshtein-based approach was originally presented by Pettersson et al. (2013b). In its basic version, no historical training data is needed, which is an important aspect considering the common data sparseness issue, as discussed in Section 1. Instead, a modern language dictionary or corpus is required, from which normalisation candidates are extracted based on edit distance comparisons to the original historical word form. If there is parallel data available, i.e. the same text in its historical and its modernised spelling, this data can be used to make more reliable Levenshtein calculations by assigning weights lower than 1 to frequently occurring edits observed in the training data. The weights are then calculated by comparing the frequency of each edit occurring in the training corpus to the frequency with which the specific source characters are left unchanged, in accordance with the following formula: $\frac{\text { Frequency of Unchanged }}{\text { Frequency of Edit }+ \text { Frequency of Unchanged }}$

Context-sensitive weights are added to handle edits affecting more than one character. The contextsensitive weights are calculated by the same formula as the single-character weights, and include the following operations:

- double deletion: personnes $\rightarrow$ persons

- double insertion: strait $\rightarrow$ straight

- single-to-double substitution: jug $e \rightarrow$ judge

- double-to-single substitution: moost $\rightarrow$ most

For all historical word forms in the training corpus that are not identical in the modern spelling, all possible single-character edits as well as multicharacter edits are counted for weighting. Hence, the historical word form personnes, mapped to the modern spelling persons, will yield weights for double-to-single deletion of -ne, as illustrated above, but also for single deletion of $-n$ and single deletion of $-e$.

Finally, a tuning corpus is used to set a threshold for which maximum edit distance to allow between the original word form and its normalisation candidate(s). Based on the average edit distance between the historical word forms and their modern spelling in the tuning corpus, the threshold is calculated by the following formula (where 1.96 times the standard deviation is added to cover $95 \%$ of the cases):

avg editdistance $+(1.96 *$ standard deviation $)$

If several normalisation candidates have the same edit distance as compared to the source word, the most frequent candidate is chosen, based on modern corpus data. If none of the highest-ranked normalisation candidates are present in the corpus, or if there are several candidates with the same frequency distribution, a final candidate is randomly chosen.

\subsection{The SMT-based Approach}

In the SMT-based approach, originally presented by Pettersson et al. (2013a), spelling normalisation is treated as a translation task. To address changes in spelling rather than full translation of words and phrases, character-based translation (without lexical reordering) is performed, a well-known technique for transliteration and 
character-level translation between closely related languages (Matthews, 2007; Vilar et al., 2007; Nakov and Tiedemann, 2012). In character-level SMT, phrases are modeled as character sequences instead of word sequences, and translation models are trained on character-aligned parallel corpora whereas language models are trained on character $\mathrm{N}$-grams.

Since the set of possible characters in a language is far more limited than the number of possible word forms, and the same corpus will present a larger quantity of character instances than token instances, only a rather small amount of parallel data is needed for training the translation models and the language models in character-based translation. Pettersson et al. (2013a) showed that with a training and tuning set of only 1,000 pairs of historical word forms mapped to modern spelling, a normalisation accuracy of $76.5 \%$ was achieved for Icelandic, as compared to $83.9 \%$ with a full-sized training corpus of 33,888 token pairs. Their full experiment on varying the size of the training data is illustrated in Figure 1.

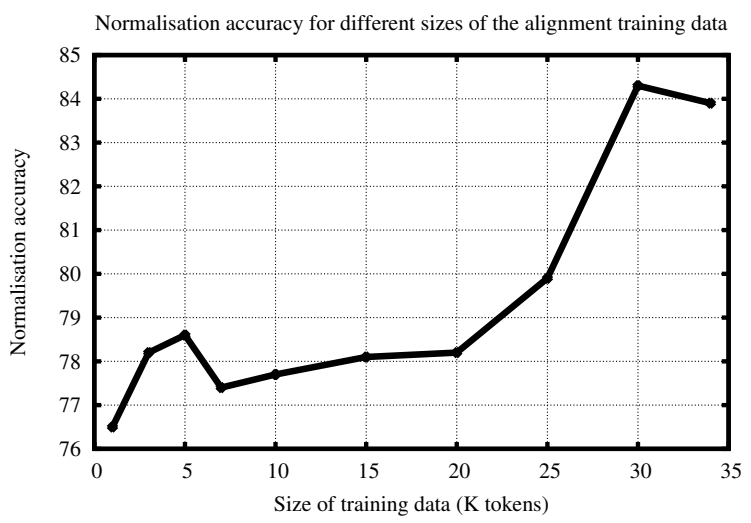

Figure 1: Normalisation accuracy when varying the size of the alignment training data.

We use the same set of training data for the SMT approach as for the filtering approach and for the assignment of weights in the Levenshtein-based approach, i.e. a set of token pairs mapping historical word forms to their manually modernised spelling. These corpora have the format of one token per line, with blank lines separating sentences. To fully adapt this format to the format needed for training the character-based translation models, the characters within each token are separated by space. The SMT system will now regard each character as a word, the full token as a sentence and the entire sentence as a section.

The SMT engine used is Moses with all its standard components. A phrase-based model is applied, where the feature weights are trained using MERT with BLEU over character-sequences as the objective function. The maximum size of a phrase (sequence of characters) is set to 10 .

Two different character alignment techniques are tested: (i) the word alignment toolkit GIZA++ (Och and Ney, 2000), and (ii) a weighted finite state transducer implemented in the $\mathrm{m} 2 \mathrm{~m}$-aligner (Jiampojamarn et al., 2007). GIZA is run with standard word alignment models for character unigrams and bigrams, whereas the $\mathrm{m} 2 \mathrm{~m}$ aligner implements transducer models based on contextindependent single character and multi-character edit operations. The transducer is trained using EM on (unaligned) parallel training data, and the final model can then be used to produce a Viterbi alignment between given pairs of character strings.

An example is given in Figure 2, where the Icelandic word forms meðr $\rightarrow$ meður and giallda $\rightarrow$ galda have been aligned at a character-level using the $\mathrm{m} 2 \mathrm{~m}$-aligner. In this example, the $\epsilon$ symbol represents empty alignments, meaning insertions or deletions. The $\epsilon$ symbol in the source word meðr denotes the insertion of $u$ in the target word meður. Likewise, the $\epsilon$ symbol in the target word galda denotes the deletion of $i$ as compared to the source word giallda. Furthermore, the alignment of giallda to galda illustrates the inclusion of multi-character edit operations, where the colon denotes a 2:1 alignment where both letters $l$ and $d$ in the source word correspond to the single letter $d$ in the target word.

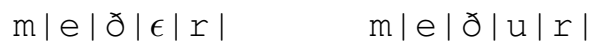

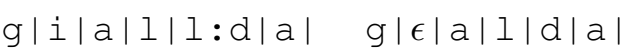

Figure 2: $\mathrm{m} 2 \mathrm{~m}$ character-level alignment.

\section{Data}

In the following, we will describe the data sets used for running the filtering approach, the Levenshtein edit distance approach, and the characterbased SMT approach for historical spelling normalisation applied to five languages: English, German, Hungarian, Icelandic, and Swedish. For convenience, we use the notions of training, tun- 
ing and evaluation corpora, which are well-known concepts within SMT. These data sets have been created by extracting every 9 th sentence from the total corpus to the tuning corpus, and every 10th sentence to the evaluation corpus, whereas the rest of the sentences have been extracted to a training corpus. ${ }^{1}$

In the filtering approach, there is in fact no distinction between training and tuning corpora, since both data sets are combined in the dictionary lookup process. As for the Levenshtein edit distance approach, the training corpus is used for extracting single-character and multi-character edits by comparing the historical word forms to their modern spelling. The edits extracted from the training corpus are then weighted based on their relative frequency in the tuning corpus.

The historical texts used for training and evaluation are required to be available both in their original, historical spelling and in a manually modernised and validated spelling. A modern translation of a historical text is generally not usable, since word order and sentence structure have to remain the same to enable training and evaluation of the proposed methods. The access to such data is very limited, meaning that the data sets used in our experiments vary in size, genres and time periods between the languages.

\subsection{English}

For training, tuning and evaluation in the English experiments, we use the Innsbruck Corpus of English Letters, a manually normalised collection of letters from the period 1386-1698. This corpus is a subset of the Innsbruck Computer Archive of Machine-Readable English Texts, ICAMET (Markus, 1999). A subset of the British National Corpus (BNC) is used as the single modern language resource both for the Levenshteinbased and for the SMT-based approach. Table 1 presents in more detail the data sets used in the English experiments.

\subsection{German}

For training, tuning and evaluation in the German experiments, we use a manually normalised subset of the GerManC corpus of German texts from the period 1650-1800 (Scheible et al., 2011). This subset contains 22 texts from the period 16591780 , within the genres of drama, newspaper text,

\footnotetext{
${ }^{1}$ For information on how to access the data sets used in our experiments, please contact the authors.
}

\begin{tabular}{|l|l|r|r|}
\hline Resource & Data & Tokens & Types \\
\hline Training & ICAMET & 148,852 & 18,267 \\
\hline Tuning & ICAMET & 16,461 & 4,391 \\
\hline Evaluation & ICAMET & 17,791 & 4,573 \\
\hline Lev. dict. & BNC & $2,088,680$ & 69,153 \\
\hline Lev. freq. & BNC & $2,088,680$ & 69,153 \\
\hline SMT lm & BNC & $2,088,680$ & 69,153 \\
\hline
\end{tabular}

Table 1: Language resources for English.

letters, sermons, narrative prose, humanities, science och legal documents. The German Parole corpus is used as the single modern language resource both for the Levenshtein-based and for the SMT-based approach (Teubert (ed.), 2003). Table 2 presents in more detail the data sets used in the German experiments.

\begin{tabular}{|l|l|r|r|}
\hline Resource & Data & Tokens & Types \\
\hline Training & GerManC & 39,887 & 9,055 \\
\hline Tuning & GerManC & 5,418 & 2,056 \\
\hline Evaluation & GerManC & 5,005 & 1,966 \\
\hline Lev. dict. & Parole & $18,662,243$ & 662,510 \\
\hline Lev. freq. & Parole & $18,662,243$ & 662,510 \\
\hline SMT lm & Parole & $18,662,243$ & 662,510 \\
\hline
\end{tabular}

Table 2: Language resources for German.

\subsection{Hungarian}

For training, tuning and evaluation in the Hungarian experiments, we use a collection of manually normalised codices from the Hungarian Generative Diachronic Syntax project, HGDS (Simon, To appear), in total 11 codices from the time period 1440-1541. The Szeged Treebank is used as the single modern language resource both for the Levenshtein-based and for the SMT-based approach (Csendes et al., 2005). Table 3 presents in more detail the data sets used in the Hungarian experiments.

\begin{tabular}{|l|l|r|r|}
\hline Resource & Data & Tokens & Types \\
\hline Training & HGDS & 137,669 & 45,529 \\
\hline Tuning & HGDS & 17181 & 8827 \\
\hline Evaluation & HGDS & 17,214 & 8,798 \\
\hline Lev. dict. & Szeged & $1,257,089$ & 144,248 \\
\hline Lev. freq. & Szeged & $1,257,089$ & 144,248 \\
\hline SMT $1 \mathrm{~m}$ & Szeged & $1,257,089$ & 144,248 \\
\hline
\end{tabular}

Table 3: Language resources for Hungarian. 


\subsection{Icelandic}

For training, tuning and evaluation in the Icelandic experiments, we use a manually normalised subset of the Icelandic Parsed Historical Corpus (IcePaHC), a manually tagged and parsed diachronic corpus of texts from the time period 1150-2008 (Rögnvaldsson et al., 2012). This subset contains four texts from the 15th century: three sagas (Vilhjálm's saga, Jarlmann's saga, and Ector's saga) and one narrative-religious text (Miðaldacevintýri). As a dictionary for Levenshtein calculations we use a combination of Beygingarlýsing Íslensks Nútímamáls, BÍN (a database of modern Icelandic inflectional forms (Bjarnadóttir, 2012)), and all tokens occurring 100 times or more in the Tagged Icelandic Corpus of Contemporary Icelandic texts, MíM (Helgadóttir et al., 2012). ${ }^{2}$ The frequency-based choice of a final normalisation candidate in the Levenshtein approach, as well as the training of a language model in the SMT approach, are done on all tokens occurring 100 times or more in the MíM corpus. Table 4 presents in more detail the data sets used in the Icelandic experiments.

\begin{tabular}{|l|l|r|r|}
\hline Resource & Data & Tokens & Types \\
\hline Training & IcePaHC & 52,440 & 9,748 \\
\hline Tuning & IcePaHC & 6,443 & 2,270 \\
\hline Evaluation & IcePaHC & 6,384 & 2,244 \\
\hline Lev. dict. & BÍ́N+MÍM & $27,224,798$ & $2,820,623$ \\
\hline Lev. freq. & MÍM & $21,339,384$ & 9,461 \\
\hline SMT $1 \mathrm{~m}$ & MÍM & $21,339,384$ & 9,461 \\
\hline
\end{tabular}

Table 4: Language resources for Icelandic.

\subsection{Swedish}

For training, tuning and evaluation in the Swedish experiments, we use balanced subsets of the Gender and Work corpus $(\mathrm{GaW})$ of court records and church documents from the time period 15271812 (Ågren et al., 2011). As a dictionary for Levenshtein calculations we use SALDO, a lexical resource developed for present-day written Swedish (Borin et al., 2008). For frequency-based choice of a final normalisation candidate, we use the Stockholm Umeå corpus (SUC) of text representative of the Swedish language in the 1990s (Ejerhed and Källgren, 1997). The SUC corpus is also used

\footnotetext{
${ }^{2}$ The BÍN database alone is not sufficient for Levenshtein calculations, since it only contains content words.
}

to train a language model in the SMT-based approach. Table 5 presents in more detail the data sets used in the Swedish experiments.

\begin{tabular}{|l|l|r|r|}
\hline Resource & Data & Tokens & Types \\
\hline Training & GaW & 28,237 & 7,925 \\
\hline Tuning & GaW & 2,590 & 1,260 \\
\hline Evaluation & GaW & 33,544 & 8,859 \\
\hline Lev. dict. & SALDO & $1,110,731$ & 723,138 \\
\hline Lev. freq. & SUC & $1,166,593$ & 97,670 \\
\hline SMT $1 \mathrm{~m}$ & SUC & $1,166,593$ & 97,670 \\
\hline
\end{tabular}

Table 5: Language resources for Swedish.

\section{Results}

Table 6 presents the results for different languages and normalisation methods, given in terms of normalisation accuracy, i.e. the percentage of tokens in the normalised text with a spelling identical to the manually modernised gold standard, and character error rate (CER), providing a more precise estimation of the similarity between the normalised token and the gold standard version at a character level. Table 7 summarises the results in terms of Precision (Pre), Recall (Rec) and F-score $(F)$ for the filtering approach, the Levenshteinbased approach (with and without filtering), and the best-performing SMT-based approach.

For the Levenshtein experiments, we have used context-sensitive weights, as described in Section 3.2. In the SMT approach, we run GIZA with standard word alignment models for character unigrams (un) and bigrams (bi). The $\mathrm{m} 2 \mathrm{~m}$ aligner is implemented with single character edit operations (1:1) and multi-character operations (2:2).

The baseline case shows the proportion of tokens in the original, historical text that already have a spelling identical to the modern gold standard spelling. In the Hungarian text, only $17.1 \%$ of the historial tokens have a modern spelling, with a character error rate of 0.85 . For German on the other hand, accuracy is as high as $84.4 \%$, with a character error rate of only 0.16. At a first glance, the historical spelling in the Hungarian corpus appears to be very similar to the modern spelling. A closer look however reveals recurrent differences involving single letter substitutions and/or the use of accents, as for fiayval $\rightarrow$ fiaival, méghalanac $\rightarrow$ meghalának and hazaba $\rightarrow$ házába. 


\begin{tabular}{|l|c|c||c|c||c|c||c|c||c|c|}
\hline & \multicolumn{2}{|c|}{ English } & \multicolumn{2}{c||}{ German } & \multicolumn{2}{c||}{ Hungarian } & \multicolumn{2}{c||}{ Icelandic } & \multicolumn{2}{c|}{ Swedish } \\
\hline & Acc & CER & Acc & CER & Acc & CER & Acc & CER & Acc & CER \\
\hline baseline & 75.8 & 0.26 & 84.4 & 0.16 & 17.1 & 0.85 & 50.5 & 0.51 & 64.6 & 0.36 \\
\hline filter & 91.7 & 0.20 & 94.6 & 0.26 & 75.0 & 0.30 & 81.7 & 0.25 & 86.2 & 0.27 \\
\hline Lev & 82.9 & 0.19 & 87.3 & 0.13 & 31.7 & 0.71 & 67.3 & 0.35 & 79.4 & 0.22 \\
\hline Lev+filter & 92.9 & 0.09 & 95.1 & 0.06 & 76.4 & 0.35 & 84.6 & 0.19 & 90.8 & 0.10 \\
\hline giza un & 94.3 & 0.07 & 96.6 & 0.04 & 79.9 & 0.21 & 71.8 & 0.30 & 92.9 & 0.07 \\
\hline giza bi & 92.4 & 0.09 & 95.5 & 0.05 & 80.1 & 0.21 & 71.5 & 0.30 & 92.5 & 0.08 \\
\hline m2m 1:1 un & 90.6 & 0.11 & 96.0 & 0.04 & 79.4 & 0.21 & 71.2 & 0.31 & 92.3 & 0.08 \\
\hline m2m 1:1 bi & 88.0 & 0.14 & 95.6 & 0.05 & 79.5 & 0.21 & 71.5 & 0.30 & 92.2 & 0.08 \\
\hline m2m 2:2 un & 90.7 & 0.11 & 96.4 & 0.04 & 77.3 & 0.24 & 71.0 & 0.31 & 91.3 & 0.09 \\
\hline m2m 2:2 bi & 87.5 & 0.14 & 95.5 & 0.05 & 79.1 & 0.22 & 71.4 & 0.31 & 92.1 & 0.08 \\
\hline
\end{tabular}

Table 6: Normalisation results given in accuracy (Acc) and character error rate (CER).

\begin{tabular}{|l|c|c|c||c|c|c||c|c|c||c|c|c||c|c|c|}
\hline & \multicolumn{3}{|c||}{ English } & \multicolumn{3}{c||}{ German } & \multicolumn{3}{c||}{ Hungarian } & \multicolumn{3}{c|}{ Icelandic } & \multicolumn{3}{c|}{ Swedish } \\
\hline & Pre & Rec & F & Pre & Rec & F & Pre & Rec & F & Pre & Rec & F & Pre & Rec & F \\
\hline filter & 93.6 & 97.8 & 95.7 & 95.0 & 99.6 & 97.2 & 77.4 & 96.0 & 85.7 & 89.3 & 90.6 & 89.9 & 87.5 & 98.3 & 92.6 \\
\hline Lev & 92.7 & 88.6 & 90.7 & 91.0 & 95.6 & 93.2 & 68.0 & 37.3 & 48.2 & 85.4 & 76.1 & 80.5 & 90.5 & 86.6 & 88.5 \\
\hline Lev+filter & 97.4 & 95.2 & 96.3 & 97.3 & 97.7 & 97.5 & 96.2 & 78.8 & 86.7 & 95.6 & 88.0 & 91.7 & 96.6 & 93.8 & 95.2 \\
\hline SMT & 98.2 & 95.9 & 97.0 & 98.7 & 97.9 & 98.3 & 98.3 & 81.3 & 89.0 & 82.0 & 85.2 & 83.6 & 98.6 & 94.1 & 96.3 \\
\hline
\end{tabular}

Table 7: Normalisation results given in precision (Pre), recall (Rec) and F-score (F).

The Icelandic corpus also has a relatively low number of tokens with a spelling identical to the modern spelling. Even though the Hungarian and Icelandic texts are older than the English, German, and Swedish texts, the rather low proportion of tokens with a modern spelling in the Icelandic corpus is rather surprising, since the Icelandic language is generally seen as conservative in spelling. A closer inspection of the Icelandic corpus reveals the same kind of subtle single letter divergences and differences in the use of accents as for Hungarian, e.g. $a d \rightarrow a ð$ and hun $\rightarrow$ hún .

The simplistic filtering approach (filter), relying solely on previously seen tokens in the training data, captures frequently occurring word forms and works surprisingly well, improving normalisation accuracy by up to 63 percentage units. The Levenshtein-based approach (Lev) in its basic version, with no parallel training data available, also improves normalisation accuracy as compared to the baseline. However, for all languages, the simplistic filtering approach yields significantly higher normalisation accuracy than the more sophisticated Levenshtein-based approach does. This could be partly explained by the fact that frequently occurring word forms have a high chance of being captured by the filtering approach, whereas the Levenshtein-based approach runs the risk of consistently normalising high-frequent word forms incorrectly. For example, in the English Levenshtein normalisation process, the high-frequent word form stonde has consistently been normalised to stone instead of stand, due to the larger edit distance between stonde and stand. The even more common word form ben, which should optimally be normalised to been, has consistently been left unchanged as ben, since the BNC corpus, which is used for dictionary lookup in the English setup, contains the proper name Ben. The issue of proper names would not be a problem if a modern dictionary were used for Levenshtein comparisons instead of a corpus, or if casing was taken into account in the Levenshtein comparisons. There would however still be cases left like stonde being incorrectly normalised to stone as described above, which would be disadvantageous to the Levenshtein-based method. The low recall figures, especially for Hungarian, also indicates that there may be old word forms that are not present in modern dictionaries and thus are out of reach for the Levenshtein-based method, as for the previously discussed Hungarian word form meghalának.

In the Lev+filter setting, the filter is used as a first step in the normalisation process. Only tokens that could not be matched through dictionary lookup based on the training corpus are normalised by Levenshtein comparisons. The idea is 
that combining these two techniques would perform better than one approach only, since highfrequent word forms are consistently normalised correctly by the filter, whereas previously unseen tokens are handled through Levenshtein comparisons. This combination does indeed perform better for all languages, and for Icelandic this is by far the most successful normalisation method of all.

For the SMT-based approach, it is interesting to note that the simple unigram models in many cases perform better than the more advanced bigram and multi-character models. We also tried adding the filter to the SMT approach, so that only tokens that could not be matched through dictionary lookup based on the training corpus, would be considered for normalisation by the SMT model. This did however not have a positive effect on normalisation accuracy, probably because the training data has already been taken care of by the SMT model, so adding the filter only led to redundant information and incorrect matches, deteriorating the results. For four out of five languages, the GIZA unigram setting yields the highest normalisation accuracy of all SMT models evaluated. For Hungarian, the GIZA bigram modell performs marginally better than the unigram model.

From the presented results, it is not obvious which normalisation approach to choose for a new language. For Icelandic, the Levenshtein-based approach combined with the filter leads to the highest normalisation accuracy. For the rest of the languages, the SMT-based approach with the GIZA unigram or bigram setting gives the best results. Generally, the Levenshtein-based method could be used for languages lacking access to annotated historical data with information on both original and modernised spelling. If, on the other hand, such data is available, the filtering approach, or the combination of filtering and Levenshtein calculations, would be likely to improve normalisation accuracy. Moreover, the effort of training a character-based SMT system for normalisation would be likely to further improve the results.

It would be interesting to also compare the results between the languages, in a language evolution perspective. This is however not feasible within the scope of this study, due to the differences in corpus size, genres and covered time periods, as discussed in Section 4.

\section{Conclusion}

We have performed a multilingual evaluation of three approaches to spelling modernisation of historical text: a simplistic filtering model, a Levenshtein-based approach and a characterbased statistical machine translation method. The results were evaluated on historical texts from five languages: English, German, Hungarian, Icelandic and Swedish. We see that all approaches are successful in increasing the proportion of tokens in the historical text with a spelling identical to the modernised gold standard spelling. We conclude that the proposed methods have the potential of enabling us to use modern NLP tools for analysing historical texts. Which approach to choose is not clear, since the results vary for the different languages in our study, even though the SMT-based approach generally works best. If no historical training data is available, the Levenshtein-based approach could still be used, since only a modern dictionary is required for edit distance comparisons. If there is a corpus of token pairs with historical and modern spelling available, training an SMT model could however result in improved normalisation accuracy. Since the SMT models are character-based, only a rather small amount of training data is needed for this task, as discussed in Section 3.3.

We believe that our results would be of interest to several research fields. From a language evolution perspective, future research would include a thorough investigation of why certain approaches work better for some languages but not for other languages, and what the results would be if the data sets for the different languages were more similar with regard to time period, size, genre etc. The latter could however be problematic, due to data sparseness. For historians interested in using modern NLP tools for analysing historical text, an extrinsic evaluation is called for, comparing the results of tagging and parsing using modern tools, before and after spelling normalisation. Finally, the proposed methods all treat words in isolation in the normalisation process. From a language technology perspective, it would be interesting to also explore ways of handling grammatical and structural differences between historical and modern language as part of the normalisation process. This would be particularly interesting when evaluating subsequent tagging and parsing performance. 


\section{References}

Maria Ågren, Rosemarie Fiebranz, Erik Lindberg, and Jonas Lindström. 2011. Making verbs count. The research project 'Gender and Work' and its methodology. Scandinavian Economic History Review, 59(3):271-291. Forthcoming.

Alistair Baron and Paul Rayson. 2008. Vard2: A tool for dealing with spelling variation in historical corpora. In Postgraduate Conference in Corpus Linguistics, Aston University, Birmingham.

Kristín Bjarnadóttir. 2012. The Database of Modern Icelandic Inflection. In AfLaT2012/SALTMIL joint workshop on Language technology for normalisation of less-resourced languages, Istanbul, May.

Alan W. Black and Paul Taylor. 1997. Festival speech synthesis system: system documentation. Technical report, University of Edinburgh, Centre for Speech Technology Research.

Marcel Bollmann, Florian Petran, and Stefanie Dipper. 2011. Rule-based normalization of historical texts. In Proceedings of the Workshop on Language Technologies for Digital Humanities and Cultural Heritage, pages 34-42, Hissar, Bulgaria.

Marcel Bollmann. 2013. POS tagging for historical texts with sparse training data. In Proceedings of the 7th Linguistic Annotation Workshop \& Interoperability with Discourse, pages 11-18, Sofia, Bulgaria, August. Association for Computational Linguistics.

Lars Borin, Markus Forsberg, and Lennart Lönngren. 2008. Saldo 1.0 (svenskt associationslexikon version 2). Språkbanken, University of Gothenburg.

C. Csendes, J. Csirik, T. Gyimóthy, and A. Kocsor. 2005. The Szeged Treebank. In Proceedings of the Eighth International Conference on Text, Speech and Dialogue (TSD 2005), Karlovy Vary, Czech Republic.

Eva Ejerhed and Gunnel Källgren. 1997. Stockholm Umeå Corpus. Version 1.0. Produced by Department of Linguistics, Umeå University and Department of Linguistics, Stockholm University. ISBN 91-7191-348-3.

Bo Han and Timothy Baldwin. 2011. Lexical normalisation of short text messages: Makn sens a \#twitter. In Association for Computational Linguistics, editor, Proceedings of the 49th Annual Meeting of the Association for Computational Linguistics, pages 368-378, Portland, Oregon, USA, June.

Sigrún Helgadóttir, Ásta Svavarsdóttir, Eiríkur Rögnvaldsson, Kristín Bjarnadóttir, and Hrafn Loftsson. 2012. The Tagged Icelandic Corpus (MÍM). In Proceedings of the Workshop on Language Technology for Normalisation of Less-Resourced Languages, pages 67-72.
Sittichai Jiampojamarn, Grzegorz Kondrak, and Tarek Sherif. 2007. Applying many-to-many alignments and hidden markov models to letter-to-phoneme conversion. In Proceedings of the Annual Conference of the North American Chapter of the Association for Computational Linguistics (NAACL-HLT 2007), pages 372-379, Rochester, NY, April.

Bryan Jurish. 2008. Finding canonical forms for historical German text. In Angelika Storrer, Alexander Geyken, Alexander Siebert, and KayMichael Würzner, editors, Text Resources and Lexical Knowledge: Selected Papers from the 9th Conference on Natural Language Processing (KONVENS 2008), pages 27-37. Mouton de Gruyter, Berlin.

Manfred Markus, 1999. Manual of ICAMET (Innsbruck Computer Archive of Machine-Readable English Texts). Leopold-Franzens-Universität Innsbruck.

David Matthews. 2007. Machine transliteration of proper names. Master's thesis, School of Informatics.

Preslav Nakov and Jörg Tiedemann. 2012. Combining word-level and character-level models for machine translation between closely-related languages. In Proceedings of the 50th Annual Meeting of the Association for Computational Linguistics (Volume 2: Short Papers), pages 301-305, Jeju Island, Korea, July. Association for Computational Linguistics.

F. J. Och and H. Ney. 2000. Improved statistical alignment models. pages 440-447, Hongkong, China, October.

Eva Pettersson, Beáta Megyesi, and Tiedemann Jörg. 2013a. An SMT approach to automatic annotation of historical text. In Proceedings of the NoDaLiDa 2013 workshop on Computational Historical Linguistics, May.

Eva Pettersson, Beáta Megyesi, and Joakim Nivre. 2013b. Normalisation of historical text using context-sensitive weighted Levenshtein distance and compound splitting. In Proceedings of the 19th Nordic Conference on Computational Linguistics (NoDaLiDa), May.

Paul Rayson, Dawn Archer, and Nicholas Smith. 2005. VARD versus Word - A comparison of the UCREL variant detector and modern spell checkers on English historical corpora. In Proceedings from the Corpus Linguistics Conference Series on-line $e$ journal, volume 1, Birmingham, UK, July.

Eiríkur Rögnvaldsson, Anton Karl Ingason, Einar Freyr Sigurdsson, and Joel Wallenberg. 2012. The Icelandic Parsed Historical Corpus (IcePaHC). In Proceedings of the Eight International Conference on Language Resources and Evaluation (LREC'12), Istanbul, Turkey, May. European Language Resources Association (ELRA). 
Silke Scheible, Richard J. Whitt, Martin Durrell, and Paul Bennett. 2011. A Gold Standard Corpus of Early Modern German. In Proceedings of the 5th Linguistic Annotation Workshop, pages 124-128, Portland, Oregon, USA, June. Association for Computational Linguistics.

Eszter Simon. To appear. Corpus building from Old Hungarian codices. In Katalin É. Kiss, editor, The Evolution of Functional Left Peripheries in Hungarian Syntax. Oxford University Press.

Wolfgang Teubert (ed.). 2003. German Parole Corpus. Electronic resource, Oxford Text Archive.

David Vilar, Jan-Thorsten Peter, and Hermann Ney. 2007. Can we translate letters? In Proceedings of the Second Workshop on Statistical Machine Translation, pages 33-39, Prague, Czech Republic, June. Association for Computational Linguistics. 